\title{
Linha de apoio direto ao doente psiquiátrico de um centro hospitalar de Portugal durante a pandemia COVID-19
}

\author{
Direct support line for psychiatric patients at a hospital center in \\ Portugal during the COVID-19 pandemic
}

\author{
Diana Malheiro Mota ${ }^{1}$, Ana Teresa Pereira ${ }^{1}$, Ana Samico ${ }^{1}$, Pedro Horta ${ }^{1}$, \\ Tânia Rodrigues ${ }^{1}$, Bárbara Moura ${ }^{1}$, Lúcia Ribeiro ${ }^{1}$
}

\begin{abstract}
Mota DM, Pereira AT, Samico A, Horta P, Rodrigues T, Moura B, Ribeiro L. Linha de apoio direto ao doente psiquiátrico de um centro hospitalar de Portugal durante a pandemia COVID-19 / Direct support line for psychiatric patients at a hospital center in Portugal during the COVID-19 pandemic. Rev Med (São Paulo). 2021 maio-jun.;100(3):197-203.
\end{abstract}

RESUMO: Introdução. O impacto da pandemia de COVID-19 na Saúde Mental tem vindo a ser reconhecido e destacado. Para ultrapassar as dificuldades no acesso aos cuidados da saúde durante este período têm surgido algumas estratégias, tais como a criação de Linhas de Apoio Psicológico e esta abordagem foi, assim, adotada pelo Serviço de Psiquiatria e Saúde Mental do Centro Hospitalar Vila Nova de Gaia/Espinho. O objetivo deste estudo é a caracterização sociodemográfica dos pacientes que acederam à Linha, a análise das patologias mais prevalentes e a reflexão sobre a importância da sua utilização em pacientes já acompanhados previamente por Psiquiatria. Material e Métodos. Foram registrados dados relativos a variáveis sociodemográficas e clínicas dos pacientes que recorreram à Linha e realizada uma análise estatística quantitativa e descritiva. Resultados. Dentre os 100 contatos realizados, $29 \%$ foram relativos ao agravamento de sintomatologia ansiosa e depressiva e $18 \%$ a alterações comportamentais decorrentes de exacerbação de sintomas psicóticos, havendo registos também de dúvidas relacionadas à prescrição e gestão de psicofármacos. A maioria das situações foram geridas telefonicamente com medidas psicoeducativas, $39 \%$ com recurso a ajustes terapêuticos minor e uma reduzida percentagem foi orientada para avaliação presencial. Conclusão. Os dados obtidos encontram-se de acordo com o reportado na literatura que demonstra que a sintomatologia ansiosa e depressiva são as mais prevalentes no contexto desta pandemia. É essencial que sejam dinamizadas mais estratégias por profissionais de saúde com experiência clínica e em articulação com os cuidados de saúde nos períodos de crise e que sejam realizados mais estudos para compreender de que forma este auxílio pode ser otimizado.

Palavras-chave: COVID-19; Pandemia; Sistemas de apoio psicossocial; Psiquiatria; Telepsiquiatria; Saúde mental.

\begin{abstract}
Introduction. The impact of the COVID-19 Pandemic on Mental Health has been recognized and highlighted. To overcome the difficulties in accessing health care during this period, some strategies have emerged, such as the creation of Psychological Support Lines and this approach was thus adopted by the Psychiatry and Mental Health Service of the Centro Hospitalar Vila Nova de Gaia/Espinho. The objective of this study is the sociodemographic characterization of the patients who accessed the Line, the analysis of the most prevalent pathologies and the reflection on the importance of its use in patients previously monitored by Psychiatry. Materials and Methods. Data on sociodemographic and clinical variables of patients who used the Line were registered and a quantitative and descriptive statistical analysis was performed. Results. Among the 100 contacts made, 29\% corresponded to worsening anxious and depressive symptoms, and $18 \%$ were related to behavioral changes resulting from exacerbation of psychotic symptoms, with records also of questions related to the prescription and management of psychiatric drugs. Most situations were managed by telephone with psychoeducational measures, $39 \%$ using minor therapeutic adjustments and a small percentage was oriented for face-to-face assessment. Conclusion. The data obtained is consonant with the literature, which refers to anxious and depressive symptoms as the most prevalent in the context of this Pandemic. It is essential that more strategies are developed by healthcare professionals with clinical experience and in cooperation with primary care services during times of crisis and that further studies are carried out to understand how this assistance can be optimized.
\end{abstract}

Keywords: COVID-19; Pandemics; Psychosocial support systems; Psychiatry; Telepsychiatry; Mental health.

1. Serviço de Psiquiatria e Saúde Mental, Centro Hospitalar Vila Nova de Gaia/Espinho, Vila Nova de Gaia, Portugal. ORCID: Pereira AT - https:// orcid.org/0000-0001-6681-6166; Mota DM - https://orcid.org/0000-0002-5697-3050; Samico A- https://orcid.org/0000-0002-0970-0593; Horta P https://orcid.org/0000-0002-1915-3838; Rodrigues T - https://orcid.org/0000-0002-3395-0008; Moura B - https://orcid.org/0000-0002-3916-6169; Ribeiro L - https://orcid.org/0000-0002-6233-0721. E-mail: ana.teresa.pereira@chvng.min-saude.pt, diana.mota@chvng.min-saude.pt, ana.samico. goncalves@chvng.min-saude.pt, pedro.horta@chvng.min-saude.pt, tania.sousa.rodrigues@chvng.min-saude.pt, barbara.moura@chvng.min-saude. pt, lucia.ribeiro@chvng.min-saude.pt.

Endereço para correspondência: Ana Teresa Pereira. Serviço de Psiquiatria e Saúde Mental, Centro Hospitalar Vila Nova de Gaia/Espinho. R. Conceição Fernandes S/N, 4434-502 - Vila Nova de Gaia, Portugal. E-mail: ana.teresa.pereira@chvng.min-saude.pt 


\section{INTRODUÇÃO}

A atual pandemia da COVID-19 motivou alterações profundas na vida da maioria dos indivíduos com implicações desde o quotidiano familiar à dinâmica laboral.

As constantes alterações de rotinas, a necessidade de adaptação a novas formas de estar e interagir aliadas ao medo de ser contagiado e às medidas de restrição, nomeadamente ao isolamento e ao afastamento social, são fatores de risco reconhecidos para o desenvolvimento ou agravamento de morbilidades psiquiátricas e psicológicas ${ }^{1}$. Por outro lado, as limitações impostas pelo novo coronavírus implicaram grandes modificações e adaptações dos serviços de saúde ${ }^{2}$. Considerando o impacto da pandemia na saúde mental da população, bem como as dificuldades de acesso aos serviços, várias estratégias foram delineadas política e institucionalmente por todo o mundo para ultrapassar tais dificuldades ${ }^{2,3}$. Uma das estratégias mais adotadas foi o recurso à teleconsulta e a criação de linhas de apoio, entre as quais as linhas de apoio psicológico ${ }^{4,5}$.

As linhas telefônicas para apoio psicológico desempenham um papel significativo no sistema de serviços de saúde mental. A sua importância é realçada durante períodos de crise global, onde a saúde mental da população é gravemente afetada. A literatura tem relatado um aumento do uso destas linhas durante a crise econômica e períodos de catástrofe ${ }^{6}$. A comunicação social tem enfatizado o uso deste recurso no contexto da pandemia de COVID-19 e reportado a sua utilização pela população em larga escala. Em Portugal foram criadas ou disponibilizadas mais de 50 linhas de apoio psicológico. A Direção Geral de Saúde Portuguesa criou um microsite com informação útil sobre Saúde Mental em tempo de pandemia e do qual consta também informação sobre a linha de apoio psicológico do Sistema Nacional de Saúde 7 .

Apesar do indiscutível reconhecimento do seu impacto, os dados sobre os efeitos da atual pandemia COVID-19 na saúde mental são ainda preliminares. Assim, considerando a escassez de relatos na literatura científica, os dados obtidos através das linhas telefônicas nesse período em que o isolamento social inviabilizou o atendimento presencial assumem particular relevância ${ }^{6}$.

O Serviço de Psiquiatria e Saúde Mental (SPSM) do Centro Hospitalar de Vila Nova de Gaia/ Espinho (CHVNG/E) é um Serviço de Psiquiatria Geral do Norte de Portugal, que dá apoio psiquiátrico agudo e crónico a uma população de cerca de 700000 habitantes. Este Serviço criou a Linha de Apoio Direto ao Doente, para pacientes com acompanhamento em Psiquiatria nesta instituição. Este canal de comunicação esteve disponível e o atendimento foi realizado todos os dias úteis das $9 \mathrm{~h}$ às 12:30h desde o dia 22 de abril de 2020 até ao final do período de suspensão da atividade assistencial presencial.
A sua divulgação foi feita através do envio de SMS aos pacientes que tinham agendamento de consulta durante o período em que as mesmas foram suspensas. Foi assegurada por Médicos Internos de Formação Específica de Psiquiatria dos últimos anos de formação (entre o quarto e o quinto ano) em articulação e com a supervisão dos Médicos Especialistas do Serviço.

Embora não pretendesse substituir a avaliação de quadros graves que justificassem uma intervenção presencial, o recurso viabilizou atender as demandas de pacientes que apresentavam sintomatologia minor, sobretudo, de caráter ansioso. Oportunizou ainda reduzir a dificuldade na gestão das questões decorrentes do isolamento social imposto e da restrição ao acesso de cuidados de saúde.

O objetivo deste trabalho é analisar as patologias mais prevalentes que motivaram a realização do contato, caracterizando sociodemograficamente os usuários, além de refletir sobre a importância da utilização do recurso nos cuidados de saúde mental dos pacientes já acompanhados previamente em Psiquiatria.

\section{MATERIAL E MÉTODOS}

O presente estudo é de natureza observacional, transversal, descritivo e de abordagem quantitativa.

A SMS enviada aos pacientes informava da sua existência, horário de funcionamento e possibilidade de contato para qualquer dúvida referente ao seu apoio psiquiátrico no Serviço.

Durante o período de funcionamento da Linha de Apoio Direto ao Doente do SPSM do CHVNG/E (de 22 de abril a 03 de julho de 2020) foram registradas as variáveis clínicas e demográficas de todos os pacientes que a contactavam numa base de dados criada para o efeito. A mesma englobava a data de contato, a identificação do paciente e número de processo, motivo principal da chamada, orientação clínica, e notas detalhando ajustes terapêuticos efetuados ou medidas psicoterapêuticas propostas. Aquando do contato telefônico, a entrevista decorria de forma livre, de acordo com o motivo que levava o paciente a procurar atendimento e consoante as suas necessidades e os dados foram recolhidos após o término do contato. Não foram aplicados guias de entrevista estruturados ou instrumentos padronizados. $\mathrm{O}$ acesso ao processo clínico do paciente foi realizado no momento do contato de forma a registar o mesmo como ato clínico para o qual cada paciente deu o seu consentimento verbal. Todas as chamadas foram atendidas por Médicos Internos de Psiquiatria entre o quarto e o quinto ano de formação.

O tratamento estatístico dos dados foi realizado com recurso ao programa IBM SPSS Statistics.

A análise descritiva dos dados incluiu o cálculo de frequências absolutas e relativas de cada uma das variáveis categóricas consideradas. No que diz respeito às 
variáveis numéricas, foi calculada a média e desvio padrão das variáveis contínuas, tal como a idade dos pacientes. Estas variáveis foram apresentadas sob a forma de tabela (Tabela 1). O número de contatos por dia foi considerado uma variável numérica discreta, sendo para esta realizado também o cálculo de frequências relativas e absolutas, e apresentado sob a forma de gráfico (Figura 1).

A correlação entre variáveis categóricas foi testada através do teste exato de Fisher (dado não ser cumprida a assunção para o teste de Chi-quadrado de existirem menos de $20 \%$ de células com valor esperado inferior a 5). São apresentados os valores de $p$ considerando um intervalo de confiança de $95 \%$.

\section{RESULTADOS}

Durante 49 dias úteis foram efetuados 100 contatos com a Linha de Apoio Direto ao Doente do SPSM do CHVNG/E, dinamizada por seis Médicos Internos de formação específica de Psiquiatria entre o quarto e o quinto ano de formação. Estes 100 contatos corresponderam a 100 chamadas, algumas realizadas pelo mesmo paciente.

Foram realizados em média dois contatos por dia, variando de zero a seis atendimentos efetuados. (Figura 1). O maior número de registos ocorreu entre o dia 22 de abril e o dia 31 de maio (69\% dos contatos, correspondendo a um total de 69 chamadas). Desde o dia 1 de junho e até ao dia 3 de julho, foram registadas 31 chamadas (31\% de todos os contatos realizados).

A maioria dos indivíduos que contataram esta linha eram do sexo feminino, $76 \%(\mathrm{n}=76)$, e apresentavam uma idade média de 58 anos (Tabela 1). A maioria ( $n=55)$ tinha idade entre os 55 e os 74 anos.

O motivo clínico mais frequente para a utilização desta linha foram os sintomas ansiosos e depressivos, globalmente responsáveis por 29\% dos contatos $(\mathrm{n}=29)$. As alterações do comportamento, majoritariamente correspondendo a casos clínicos de agitação e prostração estiveram na origem de $12 \%$ $(\mathrm{n}=12)$ das chamadas. No entanto, 6\% consistiram em descompensação de patologia psiquiátrica maior com início ou agravamento de sintomatologia psicótica. Várias chamadas foram realizadas para o esclarecimento de dúvidas, nomeadamente sobre a terapêutica $(10 \%$, $\mathrm{n}=10$ ) e um número menor sobre a realização ou não de consulta presencial $(7 \%, n=7)$. Houve registo de $17 \%$ das chamadas com o objetivo de pedir renovação da terapêutica habitual. Três pacientes contactaram esta linha "sem motivo" correspondendo a contatos realizados por questões não relacionadas com patologia mental.

A maioria dos telefonemas foi realizada pelo paciente, mas $15 \%(n=15)$ foram realizados por familiares reportando descompensações psicopatológicas. Estes contatos realizados por familiares eram relativos na maioria dos casos a pacientes do sexo feminino $(n=11$, $73 \%)$ entre os 55 e os 74 anos $(n=9,60 \%)$. O motivo mais frequente foi a presença de alterações do comportamento $(\mathrm{n}=6,40 \%)$, seguido da sintomatologia psicótica $(\mathrm{n}=3$, $20 \%$ ). Os diagnósticos mais frequentes nestes casos foram as Perturbações Depressivas ( $n=4,26,7 \%)$, Perturbações de Ansiedade, Perturbação da Personalidade e Esquizofrenia (cada um correspondendo a $n=3,20 \%$ ). A orientação mais frequente nestes casos foi o ajuste terapêutico $(n=6,40 \%)$.

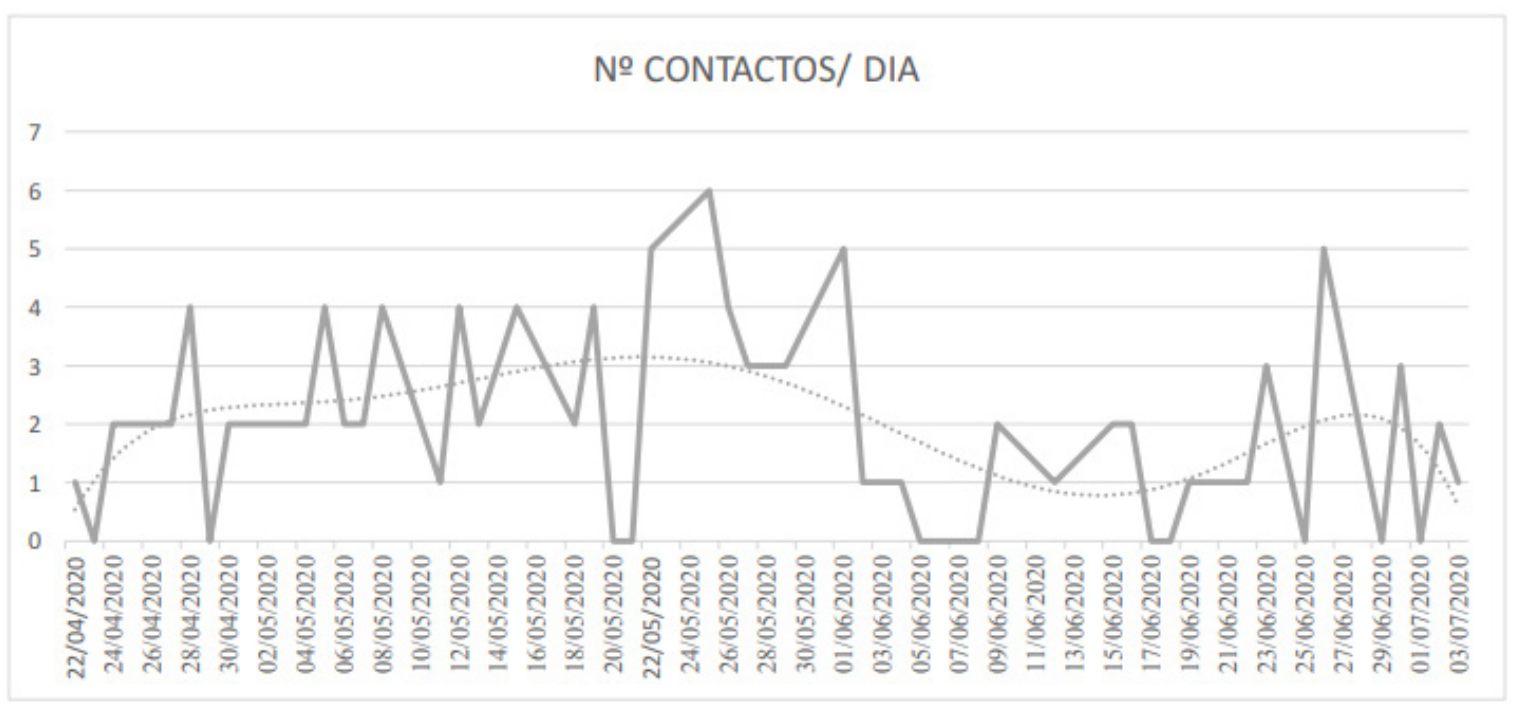

Figura 1 - Evolução do número de contatos/dia durante o funcionamento da Linha de Apoio 
Tabela 1. Caracterização sociodemográfica e clínica da amostra

\begin{tabular}{lcc}
\hline Variáveis & \\
\hline Idade (anos) & & 58,03 \\
\hline Média & 14,01 \\
Desvio padrão & 19 \\
Mínimo & 96 \\
Máximo & 60 \\
Mediana & $\mathbf{n}$ & 66 \\
Moda & & \% \\
\hline & 76 & 76 \\
\hline Sexo & 24 & 24 \\
\hline Feminino & & \\
\hline Masculino & 3 & 3 \\
Faixa etária & 2 & 2 \\
18-24 & 10 & 10 \\
$35-34$ & 22 & 22 \\
$45-54$ & 21 & 21 \\
$55-64$ & 34 & 34 \\
$65-74$ & 8 & 8 \\
$>=75$ & &
\end{tabular}

\begin{tabular}{lll}
\hline Contato realizado por & & \\
\hline Paciente & 85 & 85 \\
\hline Familiar & 15 & 15 \\
\hline
\end{tabular}

\begin{tabular}{lcc} 
Familiar & 15 & 15 \\
\hline Motivo do Contato & 16 & 16 \\
\hline Sintomatologia ansiosa & 9 & 9 \\
\hline Sintomatologia depressiva & 4 & 4 \\
\hline $\begin{array}{l}\text { Sintomatologia ansiosa } \\
\text { sintomatologia depressiva }\end{array}$ & 6 & 6 \\
\hline Insónia & 12 & 12 \\
\hline Alterações do comportamento & 6 & 6 \\
\hline Sintomatologia psicótica & 17 & 17 \\
\hline $\begin{array}{l}\text { Pedido de renovação de prescrição } \\
\text { habitual }\end{array}$ & 15 & 15 \\
\hline Dúvida sobre medicação & 7 & 7 \\
\hline Dúvida sobre consulta presencial & 4 & 4 \\
\hline Outros motivos & 4 & 4
\end{tabular}

\begin{tabular}{lll}
\hline Sem motivo & 4 & 4 \\
\hline Diagnóstico Principal & &
\end{tabular}

\begin{tabular}{lcc}
\hline Perturbação Neurocognitiva & 7 & 7 \\
\hline Perturbação de Ansiedade & 17 & 17 \\
\hline Perturbação Obsessivo-Compulsiva & 2 & 2 \\
\hline Perturbação Depressiva & 38 & 38 \\
\hline Perturbação Afetiva Bipolar & 10 & 10 \\
\hline Esquizofrenia & 7 & 7 \\
\hline Perturbação Delirante Crônica & 5 & 5 \\
\hline Psicose, sem outra especificação & 4 & 4 \\
\hline Perturbação da Personalidade & 7 & 7 \\
\hline Atraso Mental & 3 & 3 \\
\hline Orientação & & \\
\hline Psicoeducação (apenas) & 35 & 35 \\
\hline Ajuste farmacológico & 39 & 39 \\
\hline Consulta Presencial & 6 & 6 \\
\hline Serviço de Urgência & 2 & 2 \\
\hline Renovação de prescrição habitual & 17 & 17 \\
\hline Cuidados de Saúde Primários & 1 & 1 \\
\hline Total & 100 & 100 \\
\hline
\end{tabular}

Verifica-se que $38 \%$ dos atendidos encontrase em acompanhamento no SPSM do CHVNG/E com o diagnóstico de Perturbação Depressiva e 17\% com o diagnóstico de Perturbação de Ansiedade. Dezasseis indivíduos (16\%) correspondem a pacientes diagnosticados com Perturbações Psicóticas e $10 \%$ por Perturbação Afetiva Bipolar. Uma menor percentagem dos indivíduos é acompanhada por outro tipo de patologia psiquiátrica, nomeadamente Perturbação Neurocognitiva $(7 \%, n=7)$, Perturbação da Personalidade $(7 \%, n=7)$, Atraso Mental $(3 \%, n=3)$ e Perturbação ObsessivoCompulsiva $(2 \%, \mathrm{n}=2)$.

Saliente-se que nenhum dos indivíduos que contatou esta linha fez referência a contaminação com o novo coronavírus.

A Psicoeducação foi a intervenção adotada globalmente por todos os médicos e em $35 \%(n=35)$ dos contatos foram a única orientação necessária. Foram realizados ajustes farmacológicos minor em 39\% dos casos $(n=39)$, correspondentes, sobretudo, a indicações que não implicassem alterações ao esquema terapêutico prévio como a utilização de benzodiazepinas em SOS ou a sua prescrição em regime fixo. Apenas em $6 \%$ $(n=6)$ dos casos foi considerado necessário agendar uma avaliação presencial do paciente em consulta externa e dois pacientes $(2 \%)$ foram orientados para avaliação em serviço de urgência. Um paciente foi orientado para os Cuidados de Saúde Primários por já não estar em acompanhamento pelo SPSM do CHVNG/E. Em todos os casos foi dado conhecimento ao Psiquiatra Assistente que acompanhava o paciente em consulta da existência deste contacto.

Não foi encontrada relação estatisticamente significativa entre o sexo do paciente e o motivo de contato $(p=0,245)$ ou o contato ter sido realizado pelo paciente ou por familiar $(\mathrm{p}=0,752)$.

Foi encontrada uma associação estatisticamente significativa entre o sexo do paciente e o diagnóstico $(p<0,01)$, sendo que os pacientes com perturbações de ansiedade e com perturbações depressivas eram do sexo feminino em $88,2 \%$ e $94,4 \%$ dos casos, respetivamente; e entre o sexo do paciente e orientação $(p=0,043)$, sendo que dos pacientes cuja orientação foi apenas a psicoeducação, 91,4\% eram do sexo feminino. Também a faixa etária apresentava uma relação estatisticamente significativa com o sexo, estando o sexo masculino mais representado (52.4\%) na faixa dos 55 aos 64 anos que o esperado.

Não se verifica associação estatisticamente significativa entre o fato de o contato ter sido realizado pelo paciente ou por familiar e a faixa etária $(n=0,062)$, o diagnóstico $(p=0,237)$, o motivo de contato $(p=0,008)$,

Houve uma relação estatisticamente significativa entre o contato por familiar e a orientação $(p=0,02)$, nomeadamente uma maior proporção de orientação para consulta presencial (dos 6 pacientes com esta orientação, 
$\mathrm{n}=4$, ou $66,7 \%$, tiveram esta orientação).

A faixa etária apresentou uma relação estatisticamente significativa com o diagnóstico $(p<0,001)$. Os pacientes com idades entre os 65 e os 74 anos representavam uma proporção significativamente superior no grupo com diagnósticos de Perturbação Neurocognitiva (57,1\%) e Perturbações de Ansiedade $(52,9 \%)$ e Psicose SOE (100\%); a faixa etária dos 35 aos 44 anos estava significativamente sobrerepresentada no grupo com diagnóstico de Esquizofrenia $(71,4 \%)$, enquanto que no grupo com diagnóstico de Perturbação Delirante Crônica a faixa etária com proporção significativamente superior era a dos 55 aos 64 anos $(80 \%)$.

Não se verificou a existência de relação estatisticamente significativa entre a faixa etária e o motivo de contato $(p=0,105)$ ou orientação $(p=0,102)$, entre o diagnóstico e o motivo de contato $(p=0,183)$ ou orientação $(p=0,626)$ nem entre o motivo de contato e a orientação $(p=0,198)$.

\section{DISCUSSÃO}

Com este estudo exploramos os principais motivos que levaram uma amostra de pacientes acompanhada no Serviço de Psiquiatria e Saúde Mental do CHVNG/E a contatar uma Linha de Apoio Psicológico durante a pandemia de COVID-19, criada por este serviço e dinamizada por Médicos Internos de Psiquiatria nos últimos anos de formação. Identificamos ainda as principais características clínicas e sociodemográficas destes pacientes, a evolução dos quadros clínicos e as principais abordagens adotadas.

Verificamos que o principal motivo da utilização do canal de comunicação disponibilizado foi o agravamento de sintomatologia ansiosa e depressiva. A nível de diagnóstico também as Perturbações Depressivas e Ansiosas foram as mais prevalentes na amostra.

Estes dados estão de acordo com a literatura que tem descrito os sintomas ansiosos e depressivos como os mais frequentemente associados com o isolamento social, mas também com a imprevisibilidade, incerteza, gravidade e dúvidas na abordagem inerentes à pandemia de COVID-198. O isolamento social é importante para proteger a nossa saúde física, impedindo o contágio pelo vírus. Por outro lado, o seu prolongamento é fator de risco para o aparecimento de perturbações psiquiátricas. Vários estudos reportam o impacto da pandemia e das restrições associadas, não só no desenvolvimento de sintomas como humor deprimido, irritabilidade, ansiedade, medo, raiva e insônia, verificados a curto-prazo, mas também as potenciais consequências a longo prazo como o aparecimento de abuso de álcool, sintomas de perturbação de stress pós-traumático e depressão 9 .

A experiência psicológica da quarentena parece conduzir a um aumento de comportamentos de evitamento, levando a que ocorra alterações de muitos hábitos (por exemplo, evitamento de locais públicos, reforço do comportamento vigilante relativamente ao risco de infeção, etc.), reforçados através das medidas de saúde pública impostas pelos vários governos ${ }^{10}$. Durante o isolamento no período imposto pela restrição social podem imperar a tristeza, preocupação, medo, aborrecimento, frustração, culpa, desesperança, solidão e nervosismo ${ }^{2,11,12}$. A suspensão da atividade laboral e a imprevisibilidade sobre o futuro da mesma constituem fatores importantes no desenvolvimento de sintomas ansiosos e depressivos.

No período de confinamento o risco de suicídio pode ver-se aumentado pelos fatores de risco já conhecidos e por determinantes psicossociais. Durante a epidemia por SARS (Síndrome Respiratória Aguda Severa) em 2003, em Hong-Kong, verificou-se um aumento da taxa de suicídio entre a população idosa. Existem alguns relatos de casos de suicídio em contexto da infeção por SARS-CoV-2, apontando-se como fatores de risco a discriminação de que os pacientes foram alvo, motivados, muitas vezes, pela desinformação da população relativamente à doença COVID- $19^{10}$.

Existe uma grande variabilidade individual de resiliência psicológica a situações socioambientais adversas ${ }^{9}$. Neste estudo, considerando o fato de estarmos perante uma amostra de pacientes já com patologia psiquiátrica estabelecida e a realizar tratamento especializado é relevante refletirmos sobre a sua maior vulnerabilidade psicológica. Alguns estudos relatam a dificuldade no acesso ao tratamento de manutenção, durante o confinamento, por parte dos pacientes com doença mental grave, como fator de stress psicológico e de descompensação da doença psiquiátrica ${ }^{2}$. Por esse motivo, a criação de respostas alternativas como as linhas de apoio psicológico, é de suma importância.

A quarentena profilática, decretada pelos vários governos, levou milhões de pessoas em todo o mundo a ficarem em confinamento social por várias semanas. Esta é uma situação única e extraordinária, pois em nenhuma quarentena prévia imposta pela existência de outros vírus (SARS-CoV1, MERS-CoV, H1N1 e Ébola) o período de confinamento se viu tão alargado. Em Portugal, a partir do dia 12 de março, o governo iniciou medidas de contenção social e o estado de emergência no nosso país vigorou de 18 de março a 2 de maio. Para muitas pessoas, o confinamento social durou cerca de 51 dias $^{9}$.

A Linha de Apoio Direto ao Doente do SPSM do $\mathrm{CHVNG} / \mathrm{E}$ foi proposta no período inicial do confinamento, mas a sua operacionalização iniciouse apenas no final de abril. Tal demora condicionou o número total de chamadas recebidas durante uma fase crítica do período pandémico. Por outro lado, o fato da sua divulgação ter sido limitada apenas aos pacientes que tinham consulta agendada para o período abril foi 
responsável pelo número modesto de contatos diários. De fato, o período de maior número de atendimentos coincidiu com parte do período de emergência social.

A partir do final de maio verificou-se uma diminuição do número de chamadas de forma coincidente com o início do alívio das medidas de restrição, anunciadas no Plano de Desconfinamento elaborado no Conselho de Ministros no dia 29 de maio ${ }^{13}$. Esta diminuição poderá ser explicada em parte pelo desconfinamento, pelo regresso à atividade laboral e por um maior acesso à informação sobre a doença. Adicionalmente, o regresso da atividade assistencial presencial, embora condicionada, contribuiu para esta diminuição.

No final de junho verificou-se novamente um maior incremento no número de chamadas, sobretudo, correspondendo a pedidos motivados para renovação de prescrição de medicação habitual. Este aumento no período final do funcionamento da linha por motivos não relacionados com descompensação psicopatológica relaciona-se, provavelmente, com dificuldades no acesso aos cuidados de saúde primários.

A nível da abordagem e orientação, verificamos que uma reduzida percentagem das comunicações motivou orientação para avaliação presencial em consulta ou em serviço de urgência. Destaca-se que grande parte dos contatos exigiu apenas a realização de intervenções psicoeducativas. Relativamente à associação estatística significativa entre contato por familiar e maior proporção de orientação para consulta presencial, a mesma pode refletir a necessidade que os médicos sentiram de avaliar o próprio indivíduo ao invés de ouvir apenas a informação do familiar, ou da maior proporção de casos de alterações do comportamento descrita nestes contatos, carecendo de melhor caracterização.

$\mathrm{O}$ fato de a Linha ser dinamizada por Médicos Internos de Psiquiatria nos últimos anos de formação permitiu uma triagem adequada da gravidade das situações, evitando referenciações desnecessárias ao Serviço de Urgência ou duplicação de consultas presenciais numa altura em que a sua realização deveria ser restrita ao impreterivelmente necessário. Considerando o número elevado de linhas de apoio que surgiram durante a pandemia, muitas delas dinamizadas por indivíduos com escassa formação em saúde mental, é de especial importância ressalvar que apesar da sua utilidade é fundamental que estas linhas se articulem com os cuidados de saúde existentes na comunidade de modo a evitar duplicação de cuidados ${ }^{6}$.

\section{CONCLUSÕES}

A maior parte dos pacientes que utilizaram a linha de Apoio apresentavam agravamento de sintomatologia depressiva e ansiosa e uma parte significativa dos contatos relacionou-se também com pedidos de renovação de prescrição habitual ou dúvidas relacionadas com a mesma. Para além disso, a existência desta linha permitiu identificar situações associadas a psicopatologia de maior gravidade, nomeadamente alterações do comportamento e sintomatologia psicótica, possibilitando uma intervenção adequada e atempada.

O funcionamento da Linha de Apoio Direto ao Doente do SPSM do CHVNG/E abarcou parte do período de confinamento social imposto, registando-se nesta fase um maior número de contatos comparativamente ao período coincidente com o desconfinamento progressivo onde o número de chamadas foi menor. Por outro lado, a sua dinamização por Médicos Internos de Psiquiatria dos últimos anos de formação foi essencial, impedindo a duplicação de abordagens e as orientações infundadas para os Serviços de Urgência.

Neste momento, existem poucos estudos que reflitam sobre as consequências da pandemia a nível de saúde mental em pacientes previamente acompanhados em serviços de Psiquiatria, e, portanto, mais estudos serão necessários para avaliar os efeitos do confinamento impostos pela COVID-19 em pacientes com patologia mental, para além da necessidade de explorar a otimização da utilização destas linhas no auxílio dos pacientes.

Este estudo apresenta algumas limitações entre as quais as inerentes à metodologia de entrevista adotada. O enfoque na colheita de dados clínicos restritos à motivação do contato, pode ter dificultado que outras características da população em estudo fossem evidenciadas. Adicionalmente, a divulgação da Linha de Apoio ao Doente restrita aos pacientes que tinham consulta agendada no período, provavelmente, condicionou a tipologia da amostra, bem como a sua representatividade.

O tamanho reduzido da amostra também limita a possibilidade de encontrar relações estatisticamente significativas entre as variáveis estudadas, bem como a interpretação e generalização das que foram encontradas.

\section{Responsabilidades Éticas}

Conflitos de Interesse e normas éticas: Os autores declaram a inexistência de conflitos de interesse na realização do presente trabalho e declaram que foram cumpridas todas as normas de ética

Fontes de Financiamento: Não existiram fontes externas de financiamento para a realização deste artigo.

Confidencialidade dos dados: Os autores declaram ter seguido os protocolos da sua instituição acerca da publicação dos dados de pacientes.

Consentimento: Consentimento dos pacientes para publicação obtido. 
Participação dos autores: Pereira AT: concetualização do estudo, recolha e análise dos dados, redação e evisão do texto. Mota DM: concetualização do estudo, recolha e análise dos dados, redação e revisão do texto. Samico A: recolha e análise dos dados, redação e revisão do texto. Horta P: recolha e análise dos dados, redação e revisão do texto. Rodrigues $T$ : recolha e análise dos dados, redação e revisão do texto. Moura B: recolha e análise dos dados, redação e revisão do texto. Ribeiro L: concetualização do estudo, revisão do texto.

\section{REFERÊNCIAS}

1. Kar SK, Yasir Arafat SM, Kabir R, Sharma P, Saxena SK. Coping with Mental Health Challenges During COVID-19. In: Saxena SK, editor. Coronavirus disease 2019 (COVID-19): epidemiology, pathogenesis, diagnosis, and therapeutics. Singapore: Springer Singapore; 2020. p.199-213. doi: 10.1007/978-981-15-4814-7_16.

2. Li W, Yang Y, Liu ZH, Zhao YJ, Zhang Q, Zhang L, et al. Progression of Mental Health Services during the COVID-19 Outbreak in China. Int J Biol Sci. 2020;16(10):1732-8. doi: 10.7150/ijbs.45120.

3. Xiong W, Phillips MR. Translated and annotated version of the 2015-2020 National Mental Health Work Plan of the People's Republic of China. Shanghai Arch Psychiatry. 2016;28(1):4-17. doi: 10.11919/j.issn.1002-0829.216012.

4. Liu S, Yang L, Zhang C, Xiang YT, Liu Z, Hu S, et al. Online mental health services in China during the COVID-19 outbreak. Lancet Psychiatry. 2020;7(4):e17-e8. doi: 10.1016/S2215-0366(20)30077-8.

5. Zhou X, Snoswell CL, Harding LE, Bambling M, Edirippulige S, Bai X, et al. The Role of Telehealth in Reducing the Mental Health Burden from COVID-19. Telemed J E Health. 2020;26(4):377-9. doi: 10.1089/ tmj.2020.0068.

6. Peppou LE, Economou M, Skali T, Papageorgiou C. From economic crisis to the COVID-19 pandemic crisis: evidence from a mental health helpline in Greece. Eur Arch Psychiatry Clin Neurosci. 2021;271(2):407-9. doi: 10.1007/s00406-020-01165-4.
7. Portugal. Ministério da Saúde. Direção Geral de Saúde. Programa Nacional para a Saúde Mental. COVID-19. Lisboa; 2020. Disponível em: https://saudemental. covid19.min-saude.pt/.

8. Zandifar A, Badrfam R. Iranian mental health during the COVID-19 epidemic. Asian J Psychiatr. 2020;51:101990. doi: 10.1016/j.ajp.2020.101990.

9. Afonso P, Figueira L. Pandemia COVID-19: Quais são os Riscos para a Saúde Mental? Rev Portuguesa Psiquiatria Saúde Mental. 2020;6(1):2-3. doi: 10.51338/rppsm.2020. v6.i1.131.

10. Mamun MA, Griffiths MD. First COVID-19 suicide case in Bangladesh due to fear of COVID-19 and xenophobia: Possible suicide prevention strategies. Asian J Psychiatr. 2020;51:102073. doi: 10.1016/j.ajp.2020.102073.

11. Duan L, Zhu G. Psychological interventions for people affected by the COVID-19 epidemic. Lancet Psychiatry. 2020;7(4):300-2. doi: 10.1016/S2215-0366(20)30073-0.

12. Rajkumar RP. COVID-19 and mental health: a review of the existing literature. Asian J Psychiatr. 2020;52:102066. doi: 10.1016/j.ajp.2020.102066.

13. República Portuguesa. XXII Governo. Plano de Desconfinamento - Conselho de Ministros de 29 de maio de 2020. Disponível em: https://www.portugal.gov.pt/pt/gc22/ comunicacao/documento? $\mathrm{i}=$ plano-de-desconfinamentoconselho-de-ministros-de-29-de-maio-de-2020.

Submetido: 02.01.2021

Aceito: 16.06.2021 